\title{
Dacrioestenosis con dacriocistitis aguda en lactante, a propósito de un caso
}

\author{
Dacryostenosis with acute dacryocistitis in an infant, a case report \\ Mirna Paola Simón-Campos ${ }^{1, a}$, Katherine Lizbeth Bermúdez-Azañaa,a, Anderson Alexander Gonzales- \\ Rojas $^{1, a}$, Mary Carmen Vera-Abanto ${ }^{1, a}$, Tito Daniel Sevilla-Cruz ${ }^{1, a}$, Edwin Celiz-Alarcón ${ }^{2, b}$, Fernando \\ Guillermo Gil-Rodríguez ${ }^{1,2, c, d}$
}

\section{RESUMEN}

La obstrucción del conducto nasolacrimal (OCNL) o dacrioestenosis consiste en una canalización incompleta de dicho conducto. El cuadro clínico se presentó a las 2-6 semanas de edad y se caracteriza por epifora y secreción mucopurulenta. Genera diversas complicaciones, como la dacriocistitis aguda, una inflamación supurativa caracterizada por dolor, eritema e hinchazón; que puede evolucionar a un absceso lagrimal, celulitis preseptal y puede complicarse a celulitis orbitaria, absceso orbitario, trombosis del seno cavernoso, trombosis de la vena oftálmica superior y meningitis. Se presenta el caso de un lactante de siete meses 28 días de vida extrauterina con antecedente de prematuridad, diagnóstico de Tetralogía de Fallot y dacrioestenosis en ojo derecho. A su ingreso a emergencia, se evidenció eritema, aumento de calor local, secreción sero-amarillenta, consistencia dura con dolor a la palpación y edema periorbitario derecho. Clínicamente se concluyó en el diagnóstico de dacriocistitis aguda.

PALABRAS CLAVE: Conducto nasolacrimal, dacriocistitis, lactante. (Fuente: DeCS BIREME).

\section{SUMMARY}

The obstruction of the nasolacrimal conduct or dacryostenosis is the result of an incomplete canalization of such a conduct. The clinical manifestations start 2-3 weeks after birth and are characterized by epiphora and muco-purulent discharge. Dacryostenosis may lead to several complications such as acute dacrocystitis, that is a suppurative inflammation characterized by pain, erythema and swollen that can evolve to a lacrimal abscess, pre-septal cellulitis, orbital cellulitis, orbital abscess, cavernous sinus thrombosis, thrombosis of the superior ophthalmic vein and meningitis.

KEYWORDS: Nasolacrimal duct, dacryocystitis, infant. (Source: MeSH NLM).

\footnotetext{
Facultad de Medicina. Universidad Nacional de Trujillo. Trujillo, Perú.

Hospital Regional Docente de Trujillo, Trujillo, Perú.

Estudiante de Medicina del cuarto año;

Médico oftalmólogo. Retinólogo. Medico asistente;

Docente;

Médico Pediatra. Medico asistente
} 


\section{INTRODUCCIÓN}

La obstrucción del conducto nasolacrimal (OCNL) o dacrioestenosis, se produce frecuentemente por la canalización incompleta de dicho conducto en el extremo distal que origina una membrana imperforada en la válvula de Hasner, unilateral en los $2 / 3$ de los casos, afecta a aproximadamente 1,2 a $20 \%$ de los recién nacidos el $90 \%$ de los casos, se resuelve por sí solo al año ${ }^{(1,2,3,4)}$; su diagnóstico es clínico, con presencia de epífora, secreción mucopurulenta que se acumula en párpados y pestañas ${ }^{(4)}$. Sus complicaciones incluyen dermatitis, conjuntivitis, dacriocistocele, anisometropía, dacriocistitis aguda $(2,9 \%)$ o crónica (4-7).

La dacriocistitis aguda (DA) es una inflamación supurativa del saco lagrimal secundaria a la $\mathrm{OCNL}^{(8)}$. Su diagnóstico clínico se caracteriza por dolor, eritema y tumefacción, ubicado principalmente debajo del tendón cantal medial, con o sin epífora precedente ${ }^{(8,9)}$. Puede evolucionar a un absceso lagrimal y celulitis preseptal ${ }^{(9)}$. Sus complicaciones son celulitis orbitaria, absceso orbitario, trombosis del seno cavernoso, trombosis de la vena oftálmica superior y meningitis $(8,10)$.

El propósito del reporte fue presentar un caso infrecuente de dacrioestenosis que evolucionó a dacriocistitis aguda en un lactante con Tetralogía de Fallot y antecedente de prematuridad, así como las sugerencias para su manejo.

\section{PRESENTACIÓN DEL CASO}

Lactante varón de 7 meses 28 días según edad corregida y de 127 días de vida extrauterina. Nacido a las 28 semanas de edad gestacional (peso: $807 \mathrm{~g}$ y talla: $37 \mathrm{~cm}$ ), con APGAR 6 (al 1') y 8 (a los 5'), ingresó a la unidad de cuidados intensivos neonatales del Hospital Regional Docente de Trujillo (HRDT). Se realizó la valoración oftalmológica pediátrica, concluyendo en una retinopatía del prematuro grado III y recibió tratamiento único con antiangiogénico. Se confirmó clínicamente la presencia de dacrioestenosis derecha (figura 1), indicándose tratamiento conservador continuo (masaje y drenaje del conducto nasolacrimal con hisopo estéril, dexametasona más tobramicina, 1 gota cada 8 horas).

Al mes de nacido se diagnosticó tetralogía de Fallot por ecocardiografía y recibió tratamiento continuo con propanolol VO $5 \mathrm{mg}$ cada 12 horas. Ingresó al servicio

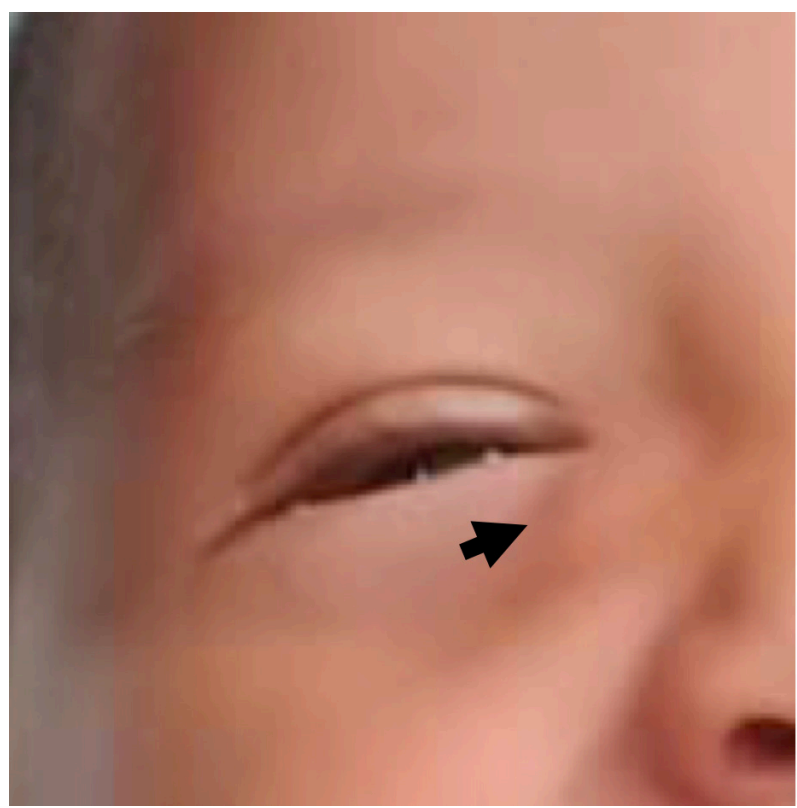

Figura 1. Lactante con dacrioestenosis derecha (flecha).

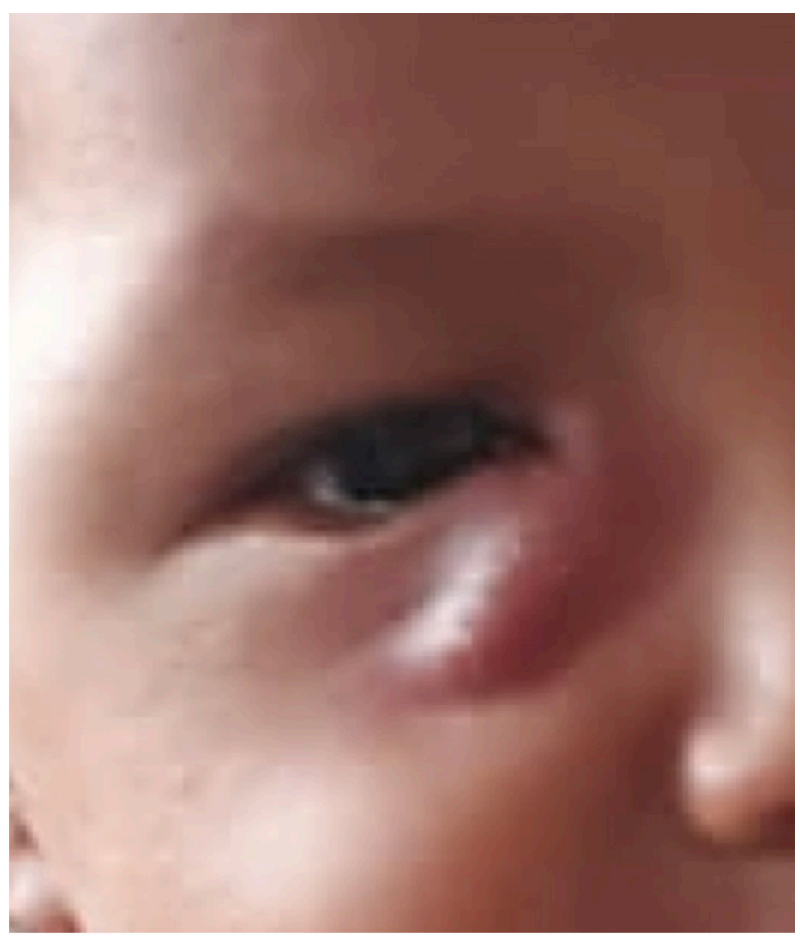

Figura 2. Lactante con diagnóstico de dacriocistitis aguda derecha con 5 días de evolución a partir de ingreso al Hospital Regional de Trujillo.

de emergencia del HRDT, con hinchazón periorbitaria derecha, coloración rojiza, caliente al tacto, que no drenaba con masajes, de dos días de evolución. Al examen físico se evidenció eritema, aumento de calor local, secreción sero-amarillenta, consistencia dura con dolor a la palpación y edema periorbitario derecho, 
postulando el diagnóstico clínico de celulitis orbitaria secundaria a dacrioestenosis y se le administró oxacilina y paracetamol. Se hospitalizó en el servicio de Medicina Pediátrica, donde de diagnosticó dacriocistitis aguda (figura 2), continuando con el tratamiento recibido en emergencia y se le agregó metamizol, tobramicina, compresas de agua tibia, presentando una evolución favorable en el transcurso de los días. Al quinto día se reemplazó la oxacilina por cefalexina vía oral y continuando con el tratamiento ya descrito. A los siete días del ingreso fue dado de alta y se encuentra en control por consultorio externo de oftalmología en HRDT.

\section{DISCUSIÓN}

El desarrollo completo del conducto nasolacrimal ocurre en el octavo mes de gestación, por ello, en prematuros, existe una canalización incompleta del conducto. Valcheva et al. ${ }^{(11)}$, analizaron 113 casos de OCNL, donde el $97 \%$ fueron niños a término, por lo que relaciona la prematuridad con disminución del riesgo de dicha afección. Por otra parte, Sathiamoorthi et al. ${ }^{(12)}$, identificaron que $189(9,5 \%)$ prematuros presentaron de manera significativa OCNL, siendo la secreción mucopurulenta, la manifestación más común. El caso presentado, se relaciona con el último estudio mencionado, ya que el infante fue prematuro y mostró secreción sero-amarillenta como rasgo principal.

El tratamiento principal de la dacrioestenosis es manejo conservador, que consiste en un masaje Crigler, masaje digital en área del saco lacrimal 2 a 3 veces al día, para romper la obstrucción membranosa; además, una limpieza de los párpados con agua tibia y uso de antibióticos tópicos para controlar el drenaje mucopurulento, disminuir el componente infeccioso y como profiláctico para la dacriocistitis $(1,5,13,14)$. En la dacriocistitis aguda, se prescriben antibióticos sistémicos empíricos, siendo los más frecuentes: ceftriaxona $(95,3 \%)$, ácido nalidíxico $(87,1 \%)$, eritromicina $(84,2 \%)$, gentamicina $(83,3 \%)$, ciprofloxacina (75,9\%), vancomicina $(67,8 \%)$ y cloranfenicol $(60,9 \%)$; que tienen acción sobre $\mathrm{S}$. aureus $(33,3 \%)$, S. epidermis $(25,6 \%)$, y S. pneumoniae $(17.9 \%)(15,16)$.

Si al año no remite la obstrucción, se requiere de manejo quirúrgico, como dacriocistorrinostomía, stent lacrimonasal o un sondaje del conducto lagrimal (4). El paciente presenta tetralogía de Fallot, lo cual junto al procedimiento quirúrgico puede traer complicaciones de alto riesgo e incluso su muerte (17). Por lo cual, se sugiere solucionar primero la tetralogía de Fallot y continuar con tratamiento conservador de la OCNL y después evaluar la necesidad de manejo quirúrgico oftálmico. La importancia del diagnóstico clínico y manejo oportuno de la dacriocistitis aguda, radica en evitar posibles complicaciones que provoquen secuelas visuales severas.

\section{Declaración de financiamiento y de conflicto de interés:}

Los autores declaramos no tener conflicto de interés, así como ninguna relación económica, personal o política que pueda influir en nuestro juicio o existir algún interés financiero que pueda constituir un conflicto de interés, ya sea real, potencial o aparente.

\section{Contribución de autoría:}

MPSC, KLBA, AAGR, MCVA, TDSC: Concepción y diseño del reporte de caso, recolección de datos del paciente, análisis e interpretación de datos; redacción del reporte; revisión crítica del artículo; aprobación de la versión final; aporte de material de estudio. ECA, FGGR: Asesoría y revisión del reporte de caso.

\section{Correspondencia:}

Mirna Paola Simón Campos.

Dirección: Mz. A21 Lt44 Manuel Arévalo II etapa -

La Esperanza.

Trujillo, Perú.

Teléfono: 51937683743.

Correo electrónico: mirnasc398@gmail.com .

\section{REFERENCIAS BIBLIOGRÁFICAS}

1. Perez Y, Patel B, Mendez M. Nasolacrimal Duct Obstruction. E Treasure Island (FL): StatPearls Publishing; 2019. (Citado el 17 de noviembre del 2019) Disponible en: https://www.ncbi.nlm.nih.gov/ books/NBK532873/?report=classic

2. Dolar A. Mode of delivery, birth weight and the incidence of congenital nasolacrimal duct obstruction. Int J Ophthalmol. 2019; 12(7):1134-1138.

3. Davies R, Watkins W, Kotecha S, Watts P. The presentation, clinical features, complications, and treatment of congenital dacryocystocele. Eye. 2017; 32(3):522-526.

4. Naranjo M, Macas C, Pacheco M, Piedrahita J. Obstrucción del conducto nasolagrimal: abordaje quirúrgico endonasal versus externo. Recimundo. 2019; 3(3):509-26. 
5. Vagge A, Ferro L, Nucci $P$, et al. Congenital Nasolacrimal Duct Obstruction (CNLDO): A Review. Diseases. 2018; 6(96):1-11.

6. Paysse E, Coats D. Congenital nasolacrimal duct obstruction (dacryostenosis) and dacryocystocele. Waltham, MA: UpToDate Inc; 2019. (Citado el 17 de noviembre del 2019) Disponible en: https://www. uptodate.com/contents/congenital-nasolacrimalduct-obstruction-dacryostenosis-and-dacryocystocele

7. Saha B, Kumari R, Sinha B. Clinical Outcome of Probing in Infants with Acute Dacryocystitis - A Prospective Study. J Clin Diagn Res. 2017; 11(8):1-3.

8. Chong K, Abdulla H, Ali M. An update on endoscopic mechanical and powered dacryocystorhinostomy in acute dacryocystitis and lacrimal abscess lagrimal. Ann Anat. 2019; 2019:151408. doi: 10.1016/j.aanat.2019.07.009

9. Ali M. Pediatric Acute Dacryocystitis. Ophthal Plast Reconstr Surg. 2015; 31(5):341-347.

10. Chandra B, Kumari R, Prasanna B. Clinical outcome of probing in infants with acute Dacryocystitis - A Prospective Study. J Clin Diagn Res. 2017; 11(8):1-3.

11. Valcheva K, Murgova S, Duhlenski B, Hristova I. Congenital nasolacrimal duct obstruction: epidemiology and risk factors. J of IMAB. 2019; 25(1):2317-2322.
12. Sathiamoorthi S, Frank R, Mohney B. Incidence and clinical characteristics of congenital nasolacrimal duct obstruction. Br J Ophthalmol. 2019; 103(4):527529. doi: 10.1136/bjophthalmol-2018-312074

13. Smaniotto A, Gazim C, Moreira H, Kormann B, Bezerra L. Congenital lacrimal obstruction: case series of probing and intubation. Rev bras oftalmol. 2016; 75(1):30-33.

14. Iqbal M, Sharif M. Comparison of nasolacrimal duct obstruction in pre-term and full-term infants. Ind J Pure App Biosci. 2019; 7(4):73-79.

15. Assefa Y, Moges F, Endris M, et al. Bacteriological profile and drug susceptibility patterns in dacryocystitis patients attending Gondar University Teaching Hospital, Northwest Ethiopia. BMC Ophthalmol. 2015; 15(1):1-8.

16. Eslami F, Ghasemi H, Moradi A, Heidari S. Microbiological study of dacryocystitis in northwest of Iran. Clin Ophthalmol. 2018; 12: 1859-1864.

17. Rodriguez T, Vidal D. Anestesia y Tetralogía de Fallot: Presentación de caso. Rev Med Multimed. 2014; 18(1):1-12.

Recibido: 19/11/2019

Aceptado: 13/09/2020 